\title{
Vasectomia laparoscópica em macacos-prego (Cebus nigritus) ${ }^{1}$
}

\author{
João P.S. Feranti ${ }^{2}$, Moira A.S. Oliveira ${ }^{3}$, Michelli W. Ataíde ${ }^{3}$, Marília T. Oliveira ${ }^{4}$, Gustavo \\ Brambatti ${ }^{5}$, Fernando V. Tomazzoni ${ }^{3}$, Wilian M. Marchezan ${ }^{4}$ e Maurício V. Brun ${ }^{6 *}$
}

\begin{abstract}
Feranti J.P.S., Oliveira M.A.S., Ataíde M.W., Oliveira M.T., Brambatti G., Tomazzoni F.V., Marchezan W.M. \& Brun M.V. 2013. [Laparoscopic vasectomy in nail-monkeys (Cebus nigritus).] Vasectomia laparoscópica em macacos-prego (Cebus nigritus). Pesquisa Veterinária Brasileira 33(7):920-923. Departamento de Clínica de Pequenos Animais, Centro de Ciências Rurais, Universidade Federal de Santa Maria, Camobi, Santa Maria, RS 97105-900, Brazil. E-mail: mauriciovelosobrun@hotmail.com

This study aimed to evaluate the technique of laparoscopic vasectomy in nail-monkeys (Cebus nigritus). Therefore eight animals were subjected to inhalation anesthesia. Three portals (two $5 \mathrm{~mm}$ and one $10 \mathrm{~mm}$ ) were positioned in the ventral abdominal wall. Vasa deferentia were isolated and cauterized by bipolar energy, and afterword sectioned near the internal inguinal ring. The procedures lasted $30.67 \pm 8.78$ minutes without complications. It could be conclude that the proposed technique is suitable for this animal species.
\end{abstract}

INDEX TERMS: Surgery, primates, nail-monkeys, Cebus nigritus, deferentectomy, videolaparoscopy.

RESUMO.- 0 presente trabalho objetivou avaliar uma técnica de vasectomia laparoscópica em macacos-prego (Cebus nigritus). Para tanto, foram utilizados oito animais submetidos à anestesia geral inalatória. Foram introduzidos três portais (dois de $5 \mathrm{~mm}$ e um de $10 \mathrm{~mm}$ ) na parede abdominal ventral. Os ductos deferentes foram isolados, cauterizados com energia bipolar e seccionados próximo ao anel inguinal interno. Os procedimentos duraram $30,67 \pm 8,78$ min. sem a ocorrência de complicações trans ou pós-operatórias o que permite concluir que a técnica proposta é adequada para essa espécie.

TERMOS DE INDEXAÇÃO: Cirurgia, primatas, macacos-prego, $\mathrm{Ce}$ bus nigritus, deferentectomia, videolaparoscopia.

\footnotetext{
${ }^{1}$ Recebido em 4 de setembro de 2012.

Aceito para publicação em 6 de maio de 2013.

${ }^{2}$ Mestrando da área de Cirurgia e Clínica Médica, Departamento de Clínica de Pequenos Animais, Centro de Ciências Rurais (CCR), Universidade Federal de Santa Maria (UFSM), Avenida Roraima 1000, Santa Maria, RS 97105-900, Brasil.

${ }^{3}$ Curso de Medicina Veterinária, Universidade de Passo Fundo, Faculdade de Agronomia e Medicina Veterinária, Campus I, Bairro São José, BR 285 Km 171, Passo Fundo, RS 99025-000, Brasil.

${ }^{4}$ Curso de Medicina Veterinária, CCR-UFSM, Avenida Roraima 1000, Santa Maria, RS 97105-900.

${ }^{5}$ Programa de Pós-Graduação em Ciências Veterinárias, Universidade Federal do Rio Grande do Sul (UFRGS), Av. Bento Gonçalves 9090, Porto Alegre, RS 91540-000, Brasil.

${ }^{6}$ Departamento de Clínica de Pequenos Animais CCR-UFSM, Avenida Roraima 1000, Santa Maria, RS 97105-900. *Autor para correspondência: mauriciovelosobrun@hotmail.com
}

\section{INTRODUÇÃO}

Os primatas do gênero Cebus apresentam ampla distribuição geográfica, estendendo-se por toda a região Neotropical, sendo notáveis por explorarem habitats diversos (Vilanova et al. 2005). Estes primatas são conhecidos por possuírem habilidades manipulativas e flexibilidade comportamental o que facilita ainda mais a sua ampla distribuição através das Américas do Sul e Central (McGrew 1998, Fragaszy et al. 2004). Os primatas são considerados onívoros, sendo sua dieta composta principalmente por frutos e insetos e, ocasionalmente, sementes e flores (De Lillo et al. 1997, Fragaszy et al. 2004). Sua maturidade sexual pode ser estimada pelo estágio de erupção dos dentes caninos e pelo peso aproximado de 2,5 kg para os machos e de 1,8 kg para as fêmeas (Nagle \& Denari 1982). Reproduzem-se muito bem e demonstram longa vida em cativeiro, podendo alcançar até 60 anos de idade (Teixeira 2003).

A vasectomia é o procedimento que consiste na interrupção da patência de ambos os ductos deferentes, podendo ser realizada em animais por diferentes técnicas a partir dos acessos convencional (Hedlund 2002, Kumar \& Raj 2012) ou laparoscópico (Wildt et al. 1981, Mahalingam et al. 2009). Em cães, a aplicação de energia elétrica no ducto deferente seguida de sua secção pelo acesso minimamente invasivo tem demonstrado efetividade, comprovada pela produção de ejaculados azoospérmicos (Wildt et al. 1981, Silva et al. 1993). A obstrução do deferente pode ser seguida ou não da secção de um segmento, na dependência da técnica escolhida (Hedlund 2002). 
Para o controle populacional de primatas do mini-zoológico da Universidade de Passo Fundo - RS (UPF), incluindo o macaco-prego (Cebus nigritus), foi indicada a realização de esterilização eletiva. Considerando as alterações de comportamento sexual e fertilidade geradas pela orquiectomia, conforme demonstrado por Sapolsky \& Krey (1988) em babuínos, a técnica mais indicada para o controle populacional de primatas é a vasectomia. Sabe-se que a produção de células da série espermatogênica é mantida após a vasectomia (Hadley \& Dym 1983, Penge et al. 2002).

Considerando a carência de relatos na literatura envolvendo vasectomia em primatas, principalmente no que se refere à aplicação de procedimentos videocirúrgicos, o presente relato descreve uma técnica laparoscópica para a realização de vasectomia em macacos-prego.

\section{MATERIAL E MÉTODOS}

Foram utilizados oito macacos-prego, machos adultos, pesando $3,16 \pm 1,05 \mathrm{~kg}$, provenientes do mini-zoológico da UPF. Promoveu-se contenção química a partir da aplicação de cloridrato de xilazina (Rompun ${ }^{\circledR}$, Lab. Bayer, São Paulo-SP) (1 mg. $\mathrm{kg}^{-1}$ ) associada à tiletamina com zolazepan (Zoletil $50^{\circledR}$, Lab. Virbac, São Paulo-SP) (3 mg.kg-1) e sulfato de atropina (Atropina ${ }^{\circledR}$, Lab. Sintofarma, São Paulo-SP) (0,03 mg.kg-1 $)$, sendo todos os fármacos mantidos na mesma seringa e administrados via IM. Após a contenção química, foi realizada ampla tricotomia abdominal. Em seguida os animais foram intubados e mantidos em anestesia geral inalatória com isoflurano vaporizado em oxigênio a 100\% em circuito semiaberto, por meio do sistema de Baraka. Realizou-se a monitorização transoperatória dos sinais vitais pela mensuração das frequências cardíaca e respiratória e saturação de oxigênio através de oximetria de pulso. Para analgesia preemptiva, os pacientes receberam flunixim meglumine (Banamine ${ }^{\circledR}$, Lab. Schering-Plough, Cotia, SP) (2mg.kg-1 IM). Aproveitou-se a oportunidade para a administração de praziquantel (Cestodan ${ }^{\circledR}$, Lab. Konig, Santana de Parnaíba-SP) (25mg. $\left.\mathrm{kg}^{-1} \mathrm{IM}\right)$, ivermectina (Ivomec ${ }^{\circledR}$, Lab. Merial, Paulínia-SP) $\left(0,2 \mathrm{mg} \mathrm{kg}^{-1} \mathrm{SC}\right)$ como método de manejo de controle parasitário. Administrou-se ainda penicilina benzatínica (Pencivet ${ }^{\circledR}$, Lab. Intervet, São Paulo-SP) (40.000 UI.kg-1 IM).

Promoveu-se incisão pré-umbilical na linha média ventral, de aproximadamente $1 \mathrm{~cm}$ para a introdução de trocáter permanente de $10 \mathrm{~mm}$ pela técnica aberta. A cavidade foi insuflada com $\mathrm{CO}_{2}$ na velocidade de $1-2 \mathrm{~L} / \mathrm{min}$ até alcançar a pressão de $10-12 \mathrm{mmHg}$. Com a utilização de endoscópio rígido de $10 \mathrm{~mm}$ e $0^{\circ}$, foram acom- panhadas as introduções de outros dois portais de $5 \mathrm{~mm}$ nas paredes abdominais laterais esquerda e direita, ambos os portais inseridos aproximadamente $3 \mathrm{~cm}$ da linha alba e $5 \mathrm{~cm}$ do primeiro portal de acesso.

Após a inspeção da cavidade, o ducto deferente foi apreendido com pinça Kelly, isolando-se os vasos deferenciais e mesoducto deferente por dissecação romba com tesoura de Metzenbaum. Os ductos foram tracionados cranialmente enquanto eram cauterizados com pinça bipolar em uma extensão de aproximadamente $1 \mathrm{~cm}$ proximalmente ao anel inguinal interno, sendo posteriormente seccionados com a tesoura no ponto médio da cauterização (Fig.1). Após verificar a ausência de hemorragias, a cavidade foi desinsuflada e a ferida de maior dimensão foi ocluída com poliglactina 910 5-0 em duas camadas (parede muscular e pele), com os padrões de Sultan e intradérmico, respectivamente. As feridas menores foram ocluídas com mesmo fio em padrão intradérmico, apenas na pele.

\section{RESULTADOS E DISCUSSÃO}

Os procedimentos cirúrgicos duraram, em média, $30,87 \pm 7,66$ minutos, com o consumo médio de $5,97 \pm 4,27 \mathrm{~L}$ de $\mathrm{CO}_{2}$. Não houve complicações trans ou pós-operatórias em nenhum animal, havendo cicatrização por primeira intenção em todos os casos. No período mínimo de 45 dias de acompanhamento, ficou demonstrada a ausência de alterações sistêmicas e cicatrização por primeira intenção, sem a necessidade de contenção para limpeza das feridas operatórias.

Sabe-se que o acesso convencional é o mais indicado/ utilizado para vasectomia nas diferentes espécies animais. Ainda que se trabalhe com técnicas minimamente invasivas, os autores do presente estudo consideram inoportuno indicar como primeira escolha a cirurgia com invasão peritoneal para a oclusão do ducto deferente, seguida ou não de secção do ducto. Contudo, torna-se necessário desenvolver procedimentos laparoscópicos para primatas, uma vez que a vasectomia laparoscópica é segura e efetiva (Wildt et al. 1981) e que pode ser requerida ou até mesmo necessária durante outro procedimento laparoscópico.

Atualmente a videolaparoscopia tem importante papel como procedimento diagnóstico, uma vez que proporciona a magnificação de imagens e possibilidade de exploração ampla das cavidades, por vezes utilizando único e diminuto acesso. Considerando a importância do uso da videocirur-

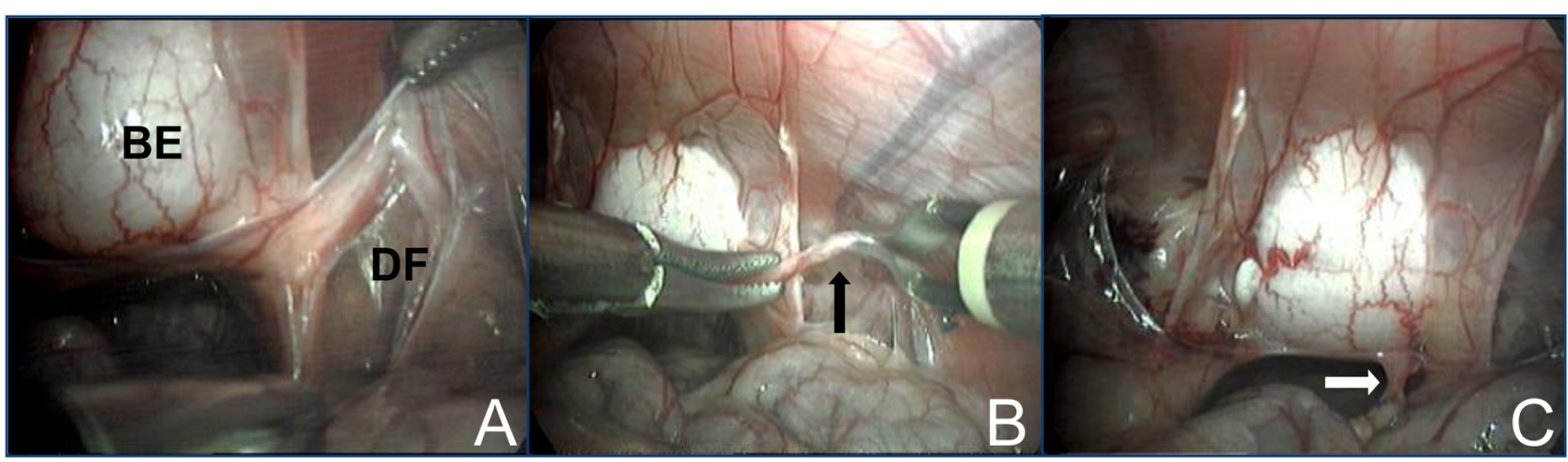

Fig.1. (A) Visualização laparoscópica do ducto deferente (DF) e bexiga (BE) durante a dissecação. (B) Cauterização do ducto deferente (seta) com pinça bipolar após prévia dissecação. (C) Visualização após a secção de ambos os ductos deferentes (seta), verifica-se que os mesmos ficaram localizados medialmente aos ligamentos laterais da bexiga. 
gia como abordagem exploratória dos órgãos abdominais, bem como para o estudo anatômico da sintopia destes, torna-se apropriada à avaliação do acesso videocirúrgico proposto com três portais e seus resultados para a presente espécie.

Nesse relato foi utilizado portal de $10 \mathrm{~mm}$ pelo serviço não disponibilizar naquele momento de óptica de menor dimensão. Condição similar ocorreu com os acessos de $5 \mathrm{~mm}$, os quais possivelmente poderiam ser substituídos por portais de $3 \mathrm{~mm}$ e instrumental compatível. Ainda assim, as operações foram executadas sem maiores dificuldades, mesmo nas cavidades dos animais menores. Localizou-se diretamente o ducto deferente pela observação do seu trajeto sobre o ligamento redondo da bexiga, condição também observada em outras espécies (Evans \& de Lahunta 1994).

A próxima etapa cirúrgica consistiu na apreensão e dissecação dos vasos deferencias junto ao ducto, de tal forma que essa última estrutura foi completamente dissecada. Essa manobra foi promovida com o intuito de evitar hemorragias durante a secção bem como para tornar necessária pouca aplicação de energia elétrica intracavitária. Cabe ressaltar a necessidade de extremo cuidado durante a cauterização, haja vista a proximidade da bexiga e dos intestinos delgado e grosso em cavidades diminutas como as observadas nos animais desse estudo. Tem-se relatado complicações laparoscópicas iatrogênicas graves devido à cauterização do cólon com posterior extravasamento de conteúdo luminal em humanos (Thompson \& Wheeless 1973), como também complicações hemorrágicas (Jansen et al. 1997, Chapron et al. 2000).

Diversas podem ser as complicações relacionadas à insuflação da cavidade com $\mathrm{CO}_{2}$, como alterações cardiocirculatórias e respiratórias (Fukushima et al. 2011). A elevação da pressão intraperitoneal e potencial absorção de $\mathrm{CO}_{2}$, condição que pode levar a aumento significativo da pressão parcial de $\mathrm{CO}_{2}$ e diminuição da pressão parcial arterial de $\mathrm{O}_{2}$ (Vannozzi et al. 2002), podem causar alterações no equilíbrio acidobásico, ventilatórias e hemodinâmicas, além de hipercapnia e acidose (Botter et al. 2005, Bergstrom et al. 2007), porém em todos os procedimentos não houve alterações mensuráveis compatíveis com as citadas.

0 tracionamento simultâneo à cauterização objetivou expor parte do ducto deferente que se encontrava posicionada através do anel inguinal, associando essa manobra à aplicação de energia elétrica próximo dessa abertura anatômica. Assim, ao promover a secção do ducto, naturalmente ocorreu afastamento de suas extremidades, sendo que a sua porção proximal à próstata se posicionou junto da bexiga e a outra se deslocou através do anel inguinal. Isso isentou a necessidade de remoção de pequeno segmento do ducto, indicada em procedimentos convencionais para prevenir uma possível manutenção de sua patência (Hedlund 2002).

Na sutura optou-se pelo uso de fio absorvível e padrão intradérmico, a fim de evitar a remoção dos pontos no pós-operatório, o que possivelmente tornaria necessária a realização de anestesia. Em primatas não humanos é indicado que se mantenham os pontos ancorados para que os pacientes não tenham acesso às suturas, evitando a manipulação e remoção prévia das mesmas.

\section{CONCLUSÕES}

0 presente relato demonstra que a técnica proposta é adequada para a realização de vasectomias laparoscópicas em macacos-prego, além de ser de fácil execução, segura e rápida.

Pela necessidade de invasão da cavidade peritoneal, a técnica pode ser indicada como procedimento secundário à outra operação laparoscópica.

0 acesso proposto permite a avaliação descritiva anatômica da sintopia e das características dos diferentes órgãos abdominais.

\section{REFERÊNCIAS}

Bergstrom M., Falk P., Park P.O. \& Holmdahl L. 2007. Peritoneal and systemic $\mathrm{pH}$ during pneumoperitoneum with $\mathrm{CO}_{2}$ and helium in a pig model. Surg. End. 22:359-364.

Botter F.C.S., Taha M.O., Fagundes D.J. \& Fagundes A.T.N. 2005. The role of pneumoperitoneum in the respiratory and hemodynamic evaluation in anaesthetized rats, with or without intubation. Revta Col. Bras. Cir. 32:261-266.

Chapron C., Pierre F., Querleu D. \& Dubuisson J.B. 2000. Major vascular complications from gynecologic laparoscopy. Gynecol. Obstet. Fertil. 28(12):880-887.

De Lillo C., Visalberghi E. \& Aversano M. 1997. The organization of exhaustive searches in a patchy space by capuchin monkeys (Cebus apella). J. Comp. Psychol. 111(1):82-90.

Evans H.E. \& De Lahunta A.M. 1994. Guia para Dissecção do Cão. $3^{\text {rd }}$ ed. Guanabara-Koogan, Rio de Janeiro. 206p.

Fragaszy D.M., VisalberghI E. \& Fedigan L.M. 2004. The Complete Capuchin: The biology of the genus Cebus. Cambridge University Press, Cambridge. 356p.

Fukushima F.B., Malm C., Andrade M.E.J., Oliveira H.P., Melo E.G., Caldeira F.M.C., Gheller V.A., Palhares M.S., Macedo S.P., Figueiredo M.S. \& Silva M.X. 2011. Cardiorespiratory and blood gas alterations during laparoscopic surgery for intra-uterine artificial inseminations in dogs. Can. Vet. J. 52:77-79.

Hadley M.A. \& Dym M. 1983. Spermatogenesis in the vasectomized monkey: quantitative analysis. Anat. Rec. 205:381-386.

Hedlund D.A. 2002. Cirurgia dos sistemas reprodutivo e genital, p.571637. In: Fossum T.W. (Ed.), Cirurgia de Pequenos Animais. $2^{2}$ ed. Roca, São Paulo.

Jansen F.W., Kapiteyn K., Trimbos-Kemper T., Hermans J. \& Trimbos J.B. 1997. Complications of laparoscopy: A prospective multicenter observation study. Brit. J. Obstet. Gynaecol. 104(5):595-600.

Kumar V. \& Raj A. 2012. No-scalpel vasectomy by eletrocauterization in free range rhesus macaques (Macaca mulatta). Open Vet. J. 2:6-9.

Mahalingam A., Kumar N., Maiti S.K., Sharma A.K., Dimri U. \& Kataria M. 2009. Laparoscopic sterilization vs. open method sterilization in dogs: a comparison of two techniques. Turk. J. Vet. Anim. Sci. 33(5): 427-436.

McGrew W.C. 1998. Culture in nonhuman primates? Annu. Rev. Anthropol. 27:301-328

Nagle C.A. \& Denari J.H. 1982. The reproductive biology of capuchin monkeys (Cebus spp). Int. Zoo Yearbook 22:143-150.

Penge B., Zhang R.D., Dai X.S., Deng X.E., Wan Y. \& Yang Z.W. 2002. Quantitative (stereological) study of the effects of vasectomy on spermatogenesis in rhesus monkeys (Macaca mulatta). Reproduction 124:847856. 
Sapolsky R.M. \& Krey L.C. 1988. Stress-induced suppression of luteinizing hormone concentration in wild baboons: Role of opiates. J. Clin. Endocrinol. Metabol. 66(4):722-6.

Silva L.D., Onclin K., Donnay I. \& Verstegen J.P. 1993. Laparoscopic vasectomy in the male dog. J. Reprod. Fertil. 47(Suppl.):399-401.

Teixeira R.H.F. 2003. Comunicação pessoal (I Curso Teórico e Prático de Clínica e Cirurgia em Primatas e Pequenos Carnívoros Selvagens, São Paulo).

Thompson B.H. \& Wheeless G.R.Jr 1973. Gastrointestinal complications of laparoscopy sterilization. Obstet. Gynecol. 41(5):669-676.
Vannozzi I., Benetti C. \& Rota A. 2002. Laparoscopic cryptorchidectomy in a cat: case report. J. Fel. Med. And Surg. 4:201-203.

Vilanova R., Júnior J.S.J., Grelle C.E.V., Marroig G. \& Cerqueira R. 2005. Limites climáticos e vegetacionais das distribuições de Cebus nigritus e Cebus robustus (Cebinae, Platyrrhini). Neotropical Primates 13(1): 14-19.

Wildt D.E., Seager S.W.J. \& Bridges C.H. 1981. Sterilization of the male dog and cat by laparoscopic occlusion of the ductus deferens. Am. J. Vet. Res. 42(11):1888-1897. 